\title{
Elaboração de tecnologia do autocuidado na prevenção de acidentes domésticos: exploração da realidade
}

\author{
Development of a self-care technology in the prevention of domestic acidentes: exploration of \\ reality \\ Elaboración de tecnología del autocuidado en la prevención de accidentes domésticos: exploración \\ de la realidad
}

Recebido: 29/08/2021 | Revisado: 12/09/2021 | Aceito: 16/09/2021 | Publicado: 17/09/2021

\author{
Mirelly de Souza Rosa \\ ORCID: https://orcid.org/0000-0001-7821-5472 \\ Universidade Federal do Maranhão, Brasil \\ E-mail: mirelly.rosa@ discente.ufma.br \\ Ana Thereza Silva dos Santos \\ ORCID: https://orcid.org/0000-0003-1944-8035 \\ Universidade Federal do Maranhão, Brasil \\ E-mail: ana.thereza@discente.ufma.br \\ Francisca Nayara dos Santos Madeira \\ ORCID: https://orcid.org/0000-0001-8838-7518 \\ Universidade Federal do Maranhão, Brasil \\ E-mail: nayara.francisca@discente.ufma.br \\ Adriana Gomes Nogueira Ferreira \\ ORCID: https://orcid.org/ 0000-0002-7107-1151 \\ Universidade Federal do Maranhão, Brasil \\ E-mail: adriana.nogueira@ufma.br \\ Ismália Cassandra Costa Maia Dias \\ ORCID: https://orcid.org/ 0000-0002-9203-0869 \\ Universidade Federal do Maranhão, Brasil \\ E-mail: ismalia.dias@ufma.br \\ Janaina Miranda Bezerra \\ ORCID: https://orcid.org/0000-0002-4799-9638 \\ Universidade Federal do Maranhão, Brasil \\ E-mail: janaina.mb@ufma.br
}

\begin{abstract}
Resumo
A pandemia pelo novo coronavírus aumentou a exposição das crianças e adolescentes aos riscos de acidentes domésticos, no entanto, também permitiu um maior contato com as tecnologias digitais sejam para fins educacionais e/ou de entretenimento. Esta nova realidade, portanto, mostrou a necessidade de disponibilizar uma tecnologia de autocuidado na prevenção de acidentes que ocorram no ambiente domiciliar. O objetivo deste trabalho foi apresentar uma revisão da literatura e a exploração da realidade para elaboração de tecnologia no autocuidado na prevenção de acidentes domésticos com foco em crianças do ensino fundamental. Trata-se de um estudo metodológico com foco nos resultados preliminares para definição do referencial teórico e a tecnologia educativa a ser adotada. Foi realizada uma revisão de literatura buscando por manuais e recomendações, como também artigos científicos que abordassem a construção e validação de tecnologias educativas sobre o tema. Para a etapa de exploração da realidade, optou-se por uma intervenção educativa com o tema "Acidentes Domésticos", aplicando-se um questionário com 120 alunos matriculados nas turmas de quarto e quinto ano do ensino fundamental, no período de março a junho de 2019 . O questionário apresentava ilustrações e era constituído por 9 perguntas objetivas. A revisão de literatura mostrou que existe uma escassez de tecnologias educativas aplicadas para este fim e que sejam direcionadas para essa faixa etária e a pesquisa exploratória da realidade mostrou que grande parte dos participantes foram acometidas por acidentes domésticos, como cortes e queimaduras, e que uma grande parcela utilizou cuidados inapropriados. Realizar revisão de literatura, conhecer a realidade associada a experiências das pesquisadoras são etapas fundamentais e contribuem na criação de uma tecnologia educativa que atenda as reais necessidades do público alvo.
\end{abstract}

Palavras-chave: Acidentes domésticos; Tecnologia educacional; Criança; Adolescente.

\begin{abstract}
The pandemic caused by the new coronavirus has increased the exposure of children and adolescentes to the risks of domestic accidents; however, it has also enabled a greater contact with digital technologies for educational purposes and/or entertainment. This work was intended to introduce a literature review and the exploration of reality for the
\end{abstract}


preparation of a technology in self-care in the prevention of domestic accidents, with a focus on elementary school children. This is a methodological study focused on preliminary results for the definition of the theoretical framework and the educational technology to be adopted. A literature review was conducted by searching for manuals and recommendations, as well as scientific articles, which addressed the construction and validation of educational technologies on the topic. For the reality exploration stage, it was decided to carry out an educational intervention with the theme "Domestic Accidents", applying a questionnaire with 120 students enrolled in fourth and fifth grade classes of elementary school, during the period from March to June 2019. The questionnaire featured illustrations and consisted of 9 objective questions. The literature review showed that there is a scarcity of educational technologies applied for this purpose and directed to this age group, while the exploratory reality research showed that a large portion of the participants were affected by domestic accidents, such as cuts and burns, and that a large portion used inappropriate care. Conducting a literature review and getting to know the reality associated with the experiences of the researchers are fundamental steps and contribute to the development of an educational technology that meets the real needs of the target audience.

Keywords: Domestic accidents; Educational technology; Child; Adolescent.

\section{Resumen}

La pandemia por el nuevo coronavirus aumentó la exposición de los niños y adolescentes a los riesgos de accidentes domésticos, sin embargo, también permitió un mayor contacto con las tecnologías digitales para fines educativos y/o entretenimiento. El objetivo de este trabajo fue presentar una revisión de la literatura y la exploración de la realidad para la elaboración de tecnología en el autocuidado para la prevención de accidentes domésticos con foco en niños de enseñanza primaria. Se trata de un estudio metodológico con foco en los resultados preliminares para la definición del referencial teórico y de la tecnología educativa a ser adoptada. Fue realizada una revisión de literatura buscando en manuales y recomendaciones, así como en artículos científicos que abordasen la construcción y validación de tecnologías educativas sobre el tema. Para la etapa de exploración de la realidad, se optó por una intervención educativa con el tema "Accidentes Domésticos", aplicándose un cuestionario con 120 alumnos matriculados en los cursos de cuarto y quinto año de enseñanza primaria, periodo de marzo a junio de 2019. El cuestionario presentaba ilustraciones y estaba constituido por 9 preguntas objetivas. La revisión de literatura mostró que existe una escasez de tecnologías educativas aplicadas para este fin y que estén dirigidas a esta franja de edad y la investigación exploratoria de la realidad mostró que gran parte de los participantes fueron afectados por accidentes domésticos, como cortes y quemaduras, y que una gran parcela utilizó cuidados inapropiados. Realizar revisión de literatura y conocer la realidad asociada a experiencias de las investigadoras son etapas fundamentales que contribuyen para la creación de una tecnología educativa que atienda las necesidades reales del público objetivo.

Palabras clave: Accidentes domésticos; Tecnología educacional; Niño; Adolescente.

\section{Introdução}

As lesões não intencionais representam as maiores causas de morbimortalidade na infância. No Brasil, as principais causas são os acidentes de trânsito, queimaduras, afogamentos, quedas, intoxicações, traumatismos e sufocação. Contudo, cerca de $90 \%$ dos casos poderiam ser evitados por meio de medidas de prevenção, sejam elas educacionais, legislativas e/ou ambientais (Gonçalves et al., 2019).

O surgimento do novo Coronavírus (SARS-CoV-2) ocasionou a necessidade da implantação de medidas de controle e prevenção rígidas devido a sua alta transmissibilidade, tais como a adoção do isolamento social, fechamento do comércio e escolas (Brasil, 2020). Essa nova realidade trouxe maior exposição das crianças e adolescentes aos riscos domésticos, uma vez que as mesmas passaram a permanecer em casa por todo período de tempo e simultaneamente ocasionou o uso mais frequente das tecnologias digitais tanto para fins educacionais, conhecimento ou diversão (Marinelli et al., 2020).

A educação é um dos meios essenciais para a promoção da saúde, cerca de $90 \%$ das lesões não intencionais podem ser evitadas por meio de medidas preventivas (Gonçalves et al., 2019). Desse modo, a educação em saúde é vista como um importante instrumento para a prevenção e promoção de saúde, em especial para as crianças, pois forja conhecimentos claros e concisos para o completo desenvolvimento dos mesmos (Paes, 2017).

As tecnologias de Informação e Comunicação - TICs surgiram para aprimorar o processo de educação e cuidado da saúde, elas englobam aparelhos eletrônicos como computadores e celulares com acesso à internet, ampliando a disseminação da informação por meio de um processo interativo. O desafio nos tempos atuais é incorporar as informações relacionadas à saúde entre as crianças e adolescentes por meio dessas tecnologias, provocando reflexões e discussão sobre o assunto (Pinto et 
al., 2017).

A construção de uma tecnologia educativa ocorre em cinco etapas, a primeira etapa consiste em um levantamento bibliográfico, no qual são feitas buscas em manuais, publicações do Ministério da Saúde, trabalhos publicados em bases de dados, teses e livros sobre a temática. Em seguida, na segunda etapa deve ser realizado um diagnóstico situacional elaborado através da exploração da realidade, uma vez que é indispensável o conhecimento da realidade do ambiente, para que sejam priorizadas as necessidades da população-alvo. Por conseguinte, na terceira etapa, inicia-se a construção da tecnologia, que por sua vez possui suas próprias fases a depender do tipo de metodologia que será construída. Posteriormente, deve ser realizada a validação do material construído, no qual este deve ser submetido a uma avaliação de conteúdo aparência por juízes especialistas, além de uma consulta com o público alvo com o propósito de reafirmar a confiabilidade do instrumento produzido. E por fim, a aplicação da tecnologia (Santos., et al, 2019).

Dessa forma, o enfermeiro é o profissional que está inserido no cenário da educação em saúde, devendo efetuar ações praticadas com a população, contribuindo para qualidade de vida. Faz-se necessário que o profissional enfermeiro atue como figura de orientador ou educador em saúde desse público, desenvolvendo ações e atividades para que a população aprenda e evolua, tomando atitudes que promovam a saúde (Kirsch, 2018).

O indivíduo na faixa etária que compreende as fases de criança e transição para a adolescência é um ser que vive em constante desenvolvimento, dispondo de facilidade no manuseio e acesso aos meios tecnológicos, o que possibilita o uso dessas ferramentas nas ações de educação em saúde. Sendo assim, entende-se que o uso de tecnologias educacionais pode auxiliar na prevenção de acidentes na infância a partir do despertar do senso crítico sobre sua saúde e bem-estar (Costa et al., 2021).

Nesse sentido, a internet tem se apresentado como recurso valioso para propagação de conhecimentos, tornando-se um espaço para a utilização de tecnologias educacionais que visam o processo de ensino-aprendizagem, uma vez que tal ferramenta propicia o compartilhamento de informações em larga escala e em um curto espaço de tempo, auxiliando no processo do autocuidado, na aquisição de novos comportamentos e hábitos (Silva, Carreiro \& Mello, 2017). Nessa perspectiva, o presente estudo tem como objetivo realizar uma revisão de literatura e exploração da realidade relacionada a acidentes na infância para desenvolvimento de tecnologia educacional que possa ser utilizada por professores, pais e responsáveis.

\section{Metodologia}

Trata-se de um estudo metodológico, a qual destina-se a desenvolver ou refinar métodos de obtenção, organização ou análise de dados (Polit \& Beck, 2011), com foco nos resultados preliminares do processo de elaboração de tecnologia educacional para promoção do autocuidado na prevenção de acidentes domésticos sofridos por crianças e adolescentes em idade escolar.

Neste relato serão apresentadas as etapas de revisão da literatura e exploração da realidade, que teve como enfoque o dimensionamento dos acidentes domésticos mais frequentes no público alvo e atitudes no pós acidente, além da consulta por tecnologias educativas já publicadas na temática proposta. Essas etapas são fundamentais para justificar e sustentar o conteúdo a ser selecionado para o desenvolvimento da tecnologia educacional.

Para a revisão de Literatura foi realizada busca nas bases de dados: MEDLINE, SCOPUS, CINAHL, Web of Science, LILACS, BDEnf, utilizando os Descritores de Ciências da Saúde (DeCS) e Medical Subject Headings (MeSH) em português, inglês e espanhol: Acidentes Domésticos, Educação em Saúde e Tecnologia Educacional. Para a etapa de exploração da realidade, optou-se por uma intervenção educativa com o tema "Acidentes Domésticos" onde aplicou-se um questionário com 120 alunos entre 9 e 14 anos de idade, matriculados nas turmas do quarto e quinto ano do ensino fundamental, no período de março a junho de 2019. 
O questionário aplicado apresentava ilustrações dos acidentes domésticos mais comuns, constituído por 9 perguntas objetivas, abordando a frequência e atitudes imediatas que os alunos teriam tomado após sofrerem o acidente. Considerando as informações coletadas com os servidores da escola, direcionou-se as perguntas para os acidentes relatados com maior frequência, como queimaduras, vazamento de gás e cortes.

Os dados coletados foram analisados de forma descritiva, digitados no banco de dados do programa Microsoft Excel® e depois transformados em tabelas e gráficos. Para a interpretação dos resultados foi realizada a discussão dos principais desfechos que emergiram por meio da avaliação crítica, contextualização e comparação, evidenciando as lacunas e as implicações das pesquisas analisadas. Esse instrumento permitiu a comparação e a organização dos dados, de acordo com as suas diferenças e similaridades, os quais foram analisados criticamente e integrados.

Por meio da análise dos artigos científicos buscou-se os referenciais teóricos mais utilizados para construção de tecnologias educacionais que possuem como público-alvo crianças e adolescentes, com enfoque no autocuidado. Além da identificação dos tipos de tecnologias mais indicadas para o público infanto-juvenil.

O estudo integra as atividades do projeto de extensão "Parceria Positiva: Saúde e Escola na Promoção da Saúde" desenvolvido em escola municipal no sudoeste Maranhense, o qual está vinculado ao curso de Enfermagem da Universidade Federal do Maranhão e foi aprovado pelo Comitê de Ética em Pesquisa da Universidade Federal do Maranhão-UFMA, sob o parecer de número 1.250 .256 .

\section{Resultados}

A revisão bibliográfica apresentou diversos achados, tais como manuais, recomendações e artigos científicos, no entanto em relação a este último foram encontradas abordagens de diferentes tecnologias construídas e utilizadas como instrumento facilitador no autocuidado de crianças acerca dos acidentes domésticos. A maioria dos estudos eram relacionados a acidentes envolvendo crianças com até 5 anos de idade e na maioria das vezes direcionados para acidentes automobilísticos, tendo como principal produto tecnologias voltadas para o uso de assentos e cadeirinhas em carros de passeio. Sobre os artigos científicos analisados, as tecnologias digitais contempladas tinham como produtos a construção e/ou avaliação de tecnologias em forma de vídeos educativos, jogos interativos, aplicativos, imagens, quiosques interativos, dentre outros.

Os resultados da revisão de literatura mostraram que os referenciais teóricos adotados pela maioria dos estudos são orientados pelos referenciais da educação popular e saúde e da teoria bioecológica do desenvolvimento humano (Lemos \& Veríssimo, 2020). Em relação à construção e validação de tecnologias educativas para o público infanto-juvenil, as TICs mais indicadas foram vídeos ou jogos educativos, uma vez que promovem maior interatividade e prendem a atenção dos alunos.

Em relação ao questionário aplicado, a análise dos dados foi realizada com os alunos que preencheram corretamente as perguntas do questionário, correspondendo a 48 dos 120 escolares. Dentre essas, foi obtido um número equivalente entre a participação dos sexos feminino e masculino, a maioria tinham entre 9 e 12 anos de idade $(85,4 \%)$ e 58,4\% (28) cursaram o $5^{\circ}$ ano do ensino fundamental (Tabela 1). 
Tabela 1. Classificação por sexo, idade e turmas dos alunos de Escola Pública Municipal, Imperatriz, MA, Brasil, 2019.

\begin{tabular}{ccc}
\hline $\begin{array}{c}\text { Variáveis } \\
\text { Sexo }\end{array}$ & $\mathbf{N}$ & $\%$ \\
Feminino & 24 & 50 \\
Masculino & 24 & 50 \\
\hline Idade & & \\
(anos) & & \\
9 & 11 & 22,9 \\
10 & 20 & 41,7 \\
11 & 8 & 16,7 \\
12 & 2 & 4,1 \\
13 & 5 & 10,5 \\
14 & 2 & 4,1 \\
\hline Turma & & \\
$4^{\text {o }}$ Ano & 20 & 41,66 \\
$5^{\text {o }}$ Ano & 28 & 58,4 \\
\hline Total & 48 & 100 \\
\hline
\end{tabular}

Legenda: $\mathrm{n}=$ amostra; $\%=$ percentual por distribuição da frequência. Fonte: Dados da pesquisa (2019).

Todos os alunos relataram já terem sofrido algum acidente doméstico, $100 \%$ cortes, 98\% queimaduras e $67 \%$ presenciaram vazamento de gás na residência. Foi identificado que os acidentes por queimaduras são os que apresentam maiores erros nos cuidados (Tabela 2).

$\mathrm{Na}$ Tabela 2 pode-se evidenciar a frequência dos acidentes domésticos, neste caso, tiveram alunos que sofreram o acidente por mais de uma vez e com objetos diferentes. A tabela ainda mostrou que a maioria dos acidentes ocorreram com objetos quentes e que $59 \%$ deles utilizaram cuidados iniciais não recomendados para este tipo de injúria, como por exemplo, o uso de creme dental, manteiga, estourar bolhas ou ainda colocar gelo. 
Tabela 2. Relação de Frequência, Causa e Tratamento de queimaduras nos alunos de Escola Pública municipal, Imperatriz, MA, Brasil, 2019.

\begin{tabular}{|c|c|c|}
\hline \multicolumn{3}{|c|}{ Queimaduras } \\
\hline Variáveis & $\mathbf{N}$ & $\%$ \\
\hline Já sofreu queimadura? & $N=48$ & \\
\hline Sim & 47 & 97,9 \\
\hline Não & 1 & 2,1 \\
\hline Não lembro & 0 & 0 \\
\hline Total & 48 & 100 \\
\hline Causa & $\mathbf{N}=71$ & \\
\hline Ferro de passar & 4 & 5,7 \\
\hline Choque elétrico & 16 & 22,5 \\
\hline Objetos quentes & 35 & 49,3 \\
\hline Outros & 16 & 22,5 \\
\hline Total & 71 & 100 \\
\hline Tratamento & $\mathbf{N}=\mathbf{8 0}$ & \\
\hline Colocar creme dental & 14 & 17,5 \\
\hline Lavar com água & 19 & 23,7 \\
\hline Estourar bolhas & 9 & 11,2 \\
\hline Colocar gelo & 26 & 32,5 \\
\hline Colocar Manteiga & 5 & 6,3 \\
\hline Não sei & 7 & 8,8 \\
\hline Total & 80 & 100 \\
\hline
\end{tabular}

Legenda: $\mathrm{n}=$ amostra; \%=percentual por distribuição da frequência.

* panelas, etc ** chapinha de cabelo, descarga de motocicletas, óleo quente e fogo.

Fonte: Dados da pesquisa, 2019.

Em relação ao vazamento de gás, $32(66,7 \%)$ alunos afirmaram já terem presenciado tal situação e 6,2\% referiram não lembrar. Como procedimento imediato a ser realizado $12(60 \%)$ escolares declararam afastar-se imediatamente e pedir ajuda, contudo $20 \%$ optaram por acender a luz ou ainda fechar as janelas nessa situação.

Dos 48 participantes que alegaram já terem sofrido cortes, a maioria, 56,5\% relatou como causa mais frequente o uso de facas, seguido de lâmina de barbear (21,7\%), 13,1\% sofreram acidentes com outros materiais como (vidro, estilete e demais materiais perfurocortantes) e em uma menor proporção $(8,7 \%)$ relataram a tesoura como material. Em relação aos cuidados realizados após o acidente, 14 (63,6\%) escolares consideraram lavar o ferimento com água e sabão como tratamento correto. No entanto, $4(18,2 \%)$ selecionaram a alternativa de colocar sal ou pó de café na lesão aberta e 9,1\% marcaram não saber o que fazer ou assoprar o ferimento como conduta (Tabela 3). 
Tabela 3. Percentual de Acertos Erros quanto aos tipos de agravos dos alunos de Escola Pública municipal, Imperatriz, MA, Brasil, 2019

\begin{tabular}{ccccc}
\hline & \multicolumn{2}{c}{ Percentual de Acertos e Erros } & & \\
\hline Tipo de Agravo & Respostas Corretas & Respostas Incorretas & Não sei & Total \\
\hline N $(\%)$ & N (\%) & N (\%) \\
\hline Queimadura & $19(39,6 \%)$ & $22(45,8 \%)$ & $7(14,6 \%)$ & \\
Vazamento de gás & $43(89,6 \%)$ & $4(8,3 \%)$ & $1(2,1 \%)$ & $48(100 \%)$ \\
Cortes & $42(87,5 \%)$ & $0(0 \%)$ & $6(12,5 \%)$ & \\
\hline
\end{tabular}

Legenda: $\mathrm{n}=$ amostra; \%= percentual por distribuição da frequência.

Fonte: Dados da pesquisa (2019).

De acordo com o questionário aplicado aos escolares, a queimadura foi o agravo que mais obteve respostas incorretas, somando-se 45,8\% do total, em contrapartida com relação ao vazamento de gás obteve-se um resultado favorável representado por $89,6 \%$ de acertos. No que se refere aos cortes, embora $12,5 \%$ tenham marcado a opção como "não sei o que fazer", a maioria $(87,5 \%)$ optou pela escolha correta.

\section{Discussão}

Os acidentes domésticos consistem em um sério problema de saúde pública por estar entre as principais causas de morte e hospitalização na infância. As queimaduras, quedas e sufocação estão no ranking dos acidentes mais frequentes, e todos eles podem ser evitados por meio da prevenção (Faria et al.,2018; Gonçalves et al., 2019).

É comum os pais acreditarem que as crianças irão perceber os perigos que existem dentro do ambiente domiciliar somente quando mais velhas. Entretanto, muitos estudos sugerem que a maioria dos acidentes ocorrem entre as faixas etárias menores, que compreende a fase pré escolar ( 2 a 6 anos). Neste período elas ainda estão desenvolvendo habilidades biológicas, sociais, psicológicas e motoras que serão aprimoradas com o passar dos anos (Silva \& Fernandes, 2019).

Vale ressaltar que, com a chegada da pandemia do Sars-Cov-2 ocorreram diversas mudanças no cotidiano dos infantes. Dentre elas se destaca a necessidade de ter o distanciamento e isolamento social, desta forma, as aulas e outras atividades foram suspensas e as crianças passaram a permanecer mais tempo dentro de casa. Consequentemente, os índices de acidentes domésticos aumentaram durante a pandemia da Covid-19 (Marcheti et al., 2020). Desse modo, o uso de vídeos educativos como tecnologia facilitadora no autocuidado da criança seria interessante, uma vez que segundo a pesquisa bibliográfica é uma tecnologia atrativa e de fácil propagação por meio da internet. Dessa forma, realizar essa fase exploratória é indispensável para o desenvolvimento de uma tecnologia que atenda às reais necessidades do público alvo.

De acordo com uma pesquisa realizada pelo plano da Rede Nacional da Primeira Infância (2012), as queimaduras representam $7 \%$ dos acidentes ocorridos com crianças em âmbito doméstico. Entre as queimaduras mais frequentes estão a chama de fogo, contato com a água fervente e objetos aquecidos (Chiarelli, Salles \& Ramalho, 2019; Lucena, 2017). Na escola avaliada neste estudo ela teve uma representatividade maior e quando analisados os conhecimentos de primeiros socorros relacionados às queimaduras, observou-se que grande parte das crianças adotaram condutas incorretas, tais como colocar gelo, creme dental, estourar bolhas, colocar manteiga ou ainda não saber o que fazer, sendo que apenas $23,7 \%$ considerou lavar a queimadura com água e sabão.

No que concerne ao vazamento de gás, observou-se que a maioria dos alunos entendiam que deviam afastar-se e pedir ajuda, no entanto, houveram quantidades consideráveis (40\%) que marcaram a opção incorreta, valor somado entre respostas das alternativas de acender a luz e fechar as portas e janelas. Desse modo, o uso do gás GLP (gás liquefeito de petróleo), 
comumente conhecido como gás de cozinha é responsável por diversos acidentes no Brasil e no mundo, devido a vazamento junto ao acionamento do circuito de iluminação ou uso de algum aparelho no ambiente contaminado (Pinheiro, 2018).

As crianças correm riscos de sofrerem acidentes em qualquer lugar da casa, entretanto, a cozinha apresenta uma maior taxa por ser um ambiente cheio de utensílios e até mesmo situações que podem resultar em um incidente, dentre eles o corte com objetos perfurocortantes (Frota et al.,2019). De acordo com as respostas dos escolares em relação aos cortes, a maioria possuía conhecimento adequado sobre cuidados em casos de lesões na pele, ou seja, embora seja um dos acidentes mais frequentes, os alunos sabem a conduta adequada nesta ocasião.

As limitações deste estudo consistem na dificuldade de interpretação das perguntas do questionário, visto que, apesar de optar por uma linguagem acessível à faixa etária dos escolares, adicionando ilustrações nas alternativas e com o auxílio de um monitor para leitura, percebeu-se uma quantidade significativa de questionários incompletos e/ou com respostas dúbias. Contudo, compreende-se que é uma limitação inerente à fase infantil, uma vez que faz parte da natureza comportamental das crianças serem mais ativas, prestar menos atenção às tarefas que lhe são pedidas, sendo na maior parte dos casos um comportamento normal e atenuado naturalmente à medida do crescimento. Nesse sentido, é essencial ouvir as reais necessidades e entender melhor suas limitações (Neves \& Leal, 2018).

Compreender e identificar as causas multifatoriais e receber orientações adequadas quanto aos acidentes é a forma mais eficaz de prevenir e evitar agravos. A prevenção de um acidente pode ser realizada em três momentos: no pré evento, evento e pós evento. É fundamental ter uma maior atenção na prevenção e, caso o incidente ocorra, deve-se saber a melhor forma de agir. Nesse contexto, as ações de educação e promoção da saúde têm papel fundamental em todas as etapas do processo, além de contribuir para redução dos acidentes (Frota et al., 2019; Lima Pestana et al., 2013).

\section{Conclusão}

Realizar revisão bibliográfica, conhecer a realidade por meio de uma pesquisa exploratória e associar esses achados as experiências já vivenciadas pelas pesquisadoras por meio de um projeto de extensão desenvolvido com o público alvo favorecerá a criação de uma tecnologia de autocuidado na prevenção de acidentes domésticos que atenda as reais necessidades da população e que possa ser utilizada por professores, pais e/ou responsáveis.

Os achados demonstraram que poucos são os estudos que abordam a criação e validação de tecnologia educativa sobre acidentes domésticos para a faixa etária de 9 a 14 anos de idade, entre os tipos de tecnologias encontradas, o uso de vídeos animados e jogos educativos parecem contemplar de forma mais eficiente este tipo de público por permitirem maior entusiasmo e interatividade. A análise do estudo evidenciou que os acidentes domésticos merecem atenção, sobretudo, os cortes e queimaduras e para nortear a construção e validação desses instrumentos os referenciais da educação popular e saúde e da teoria bioecológica do desenvolvimento humano podem ser utilizados.

Tratando-se do público infanto-juvenil, as atividades educativas baseadas no uso de tecnologias em saúde são essenciais devido ao desconhecimento desse grupo de estudo a respeito da conduta adequada frente às lesões não intencionais. E como já citado, a enfermagem tem um papel fundamental nesse processo, realizando ações pautadas na sua prática e favorecendo o processo de ensino- aprendizagem.

\section{Referências}

Brasil. Ministério da Saúde. (2020). Protocolo de Tratamento Do Novo Coronavírus (2019-NCoV).” 32. www.saude.gov.br.

Castro, V. Q. D. (2019). Dental Adventure: Jogo Digital como Estratégia de Promoção de Saúde Bucal.

Chiarelli, A., Romão, D. M. M., Camargo Filho, F., Salles, I., Barreto, J. O. M., Boeira, L. D. S., \& Ramalho, W. (2019). Prevenção de acidentes domésticos no Distrito Federal. 
da Frota, K. C., de Sousa Santos, L. T., Oliveira, L. S., Marques, M. F., \& de Azevedo Ponte, K. M. (2019). Tecnologias educativas: estratégias eficientes para a promoção da saúde de idosos: estrategias eficientes para la promoción de la salud de idosos. Revista Saúde. com, 15(2).

da Costa, V. C., do Amaral Silva, K. R., de Lima Felix, L. K. C., de Lima Nascimento, M. M., \& Ferreira, E. B. (2021). Prototipação de game educativo para prevenção de acidentes na infância. Enfermagem em Foco, 12(1).

da Silva Pinheiro, A. L., \& Lourenço, F. G. (2018). Modelo de um dispositivo de segurança para detecção de vazamento do gás de cozinha com bloqueio do circuito elétrico. Projectus, 3(1), 15-35.

dos Santos, S. B., de Araújo Machado, A. P., Sampaio, L. A., de Abreu, L. C., \& Bezerra, I. M. P. (2019). Sífilis adquirida: construção e validação de tecnologia educativa para adolescentes. Journal of Human Growth and Development, 29(1), 65-74.

Echer, I. C. (2005). The development of handbooks of health care guidelines. Revista latino-americana de enfermagem, 13(5), 754-757.

Faria, C. G., Queiroz, D. B., Matias, O. M., \& Melo, T. P. (2018). Principais causas de internação por acidentes domésticos na infância em um hospital Universitário do Oeste do Paraná. Brazilian Journal of Surgery and Clinical Research, 22(2), 103-109.

Gonçalves, A. C., Araújo, M. P. B. D., Paiva, K. V. D., Menezes, C. D. S. A., Silva, A. É. M. C. D., Santana, G. D. O., \& Lourenção, P. L. T. D. A. (2019). Acidentes na infância: casuística de um serviço terciário em uma cidade de médio porte do Brasil. Revista do Colégio Brasileiro de Cirurgiões, 46.

Kirsch, G. H., \& Slob, E. M. G. B. (2018). Atuação do enfermeiro na educação em saúde da população. Revista Saúde e Desenvolvimento, 12(13), $218-233$.

Lemos, R. A., \& Veríssimo, M. D. L. Ó. R. (2020). Methodological strategies for the elaboration of educational material: focus on the promotion of preterm infants' development. Ciencia \& saude coletiva, 25, 505-518.

Lima Pestana, A., Haenscke Senna, M., Pereira do Nascimento, E. R., \& Schulter Buss Heidemann, I. T. (2013). Estratégias de promoção à saúde e a prevenção de acidentes no ambiente domiciliar: uma anàlise reflexiva. Journal of Nursing UFPE/Revista de Enfermagem UFPE, 7(11).

Marcheti, M. A., Luizari, M. R. F., Marques, F. R. B., Cañedo, M. C., Menezes, L. F., \& Volpe, I. G. (2020). Acidentes na infância em tempo de pandemia pela COVID-19. Rev. Soc. Bras. Enferm. Ped, 20(spe), 16-25.

Marinelli, N. P., Albuquerque, L. P. D. A., Sousa, I. D. B. D., Batista, F. M. D. A., Mascarenhas, M. D. M., \& Rodrigues, M. T. P. (2020). Evolução de indicadores e capacidade de atendimento no início da epidemia de COVID-19 no Nordeste do Brasil, 2020. Epidemiologia e Serviços de Saúde, 29.

Mendes, K. D. S., Silveira, R. C. D. C. P., \& Galvão, C. M. (2008). Revisão integrativa: método de pesquisa para a incorporação de evidências na saúde e na enfermagem. Texto \& contexto-enfermagem, 17, 758-764.

Neves, M., \& Leal, D. (2018). Design de interação e o desenvolvimento do jogo para crianças com perturbação de hiperatividade e défice de atenção dos 6 aos 10 anos. Convergências: Revista de Investigação e Ensino das Artes, 11, 1-10.

Paes, C. C. D. C., \& Paixão, A. N. D. P. (2017). A importância da abordagem da educação em saúde: revisão de literatura. Revista de Educação do Vale do São Francisco-REVASF, 6(11).

Pinto, A. C. S., Scopacasa, L. F., Bezerra, L. L. D. A. L., Pedrosa, J. V., \& Pinheiro, P. N. D. C. (2017). Uso de tecnologias da informação e comunicação na educação em saúde de adolescentes: revisão integrativa. Rev. enferm. UFPE on line, 634-644.

Polit, D. F., \& Beck, C. T. (2011). Fundamentos de pesquisa em enfermagem: avaliação de evidências para a prática da enfermagem. Artmed Editora.

Silva, D. M. D. L., Carreiro, F. D. A., \& Mello, R. (2017). Tecnologias educacionais na assistência de enfermagem em educação em saúde: revisão integrativa. Rev. enferm. UFPE on line, 1044-1051.

Silva, J. D. S., \& Fernandes, K. D. S. (2020). Acidentes domésticos mais frequentes em crianças. 